\title{
Eficiencia del sector ganadero valenciano: Aproximación no paramétrica
}

\author{
C. Calafat-Marzal ${ }^{*}$, M.L. Martí-Selva y R. Puertas-Medina \\ Departamento de Economía y Ciencias Sociales. Universitat Politècnica de Valencia. Camino de Vera, s/n. \\ 46002. Valencia. Spain
}

\section{Resumen}

El objetivo de este estudio es analizar la eficacia de las granjas ganaderas en la Comunidad Valenciana, en particular en el caso del porcino y las aves de engorde, que actualmente aportan el mayor peso económico al PIB agrícola de la Comunidad. Mediante la creación de una función de producción representativa de las asignaciones existentes en la granja y utilizando el Análisis Envolvente de Datos, se determinarán las granjas que alcanzan un nivel óptimo de producción en ausencia de ineficiencias. Los resultados obtenidos en este estudio confirman que los sectores porcino y avícola de la Comunidad Valenciana tienen un potencial significativo de mejora. El número de granjas situadas en el umbral de eficiencia es bajo para ambas especies, especialmente cerdos, donde solo el 5,11\% de las granjas analizadas son completamente eficientes. Una comparación de los sectores avícola y porcino revela que los problemas ambientales que rodean a la industria porcina también podrían ser una de las causas de la deficiente gestión económica.

Palabras clave: Ganadería, Análisis Envolvente de Datos, gestión de granjas, sectores de ganadería intensiva.

\section{Abstract \\ Efficiency in the Valencian pig and poultry sector: a non-parametric approximation}

The aim of this study is to analyse the efficiency of livestock farms in the Valencian community, particularly for pig and poultry, which currently are the main contributors to the agricultural GDP of the Community. By creating a production function representative of the existing allocations on farm and using Data Envelopment Analysis, the farms that reach an optimal level of production in the absence of inefficiencies will be identified. The results obtained in this study confirm that the pig and poultry sectors of the Valencian Community have significant potential for improvement. The number of farms situated at the threshold of efficiency is low for both species, especially pigs, where only $5.11 \%$ of the analysed farms are completely efficient. A comparison of the poultry and pig sectors reveals that the environmental problems surrounding the pig industry could also be one of the causes of deficient economic management.

Keywords: Livestock farming, Data Envelopment Analysis, farm management, intensive livestock sectors.

\footnotetext{
* Autor para correspondencia: macamar3@esp.upv.es https://doi.org/10.12706/itea.2018.005
} 


\section{Introducción}

En las últimas décadas, el sector ganadero se ha visto inmerso en profundos cambios debido a modificaciones legislativas, referentes tanto a su potencial contaminante como a su contribución en la seguridad alimentaria de los productos obtenidos. En este sentido, se han diseñado políticas comunitarias, nacionales y regionales, que han modificado la gestión de las explotaciones. Las normativas nacionales y regionales (Ley 6/2003, de 4 de marzo, de la Generalitat, de Ganadería de la Comunidad Valenciana (BOE, 2003)) han concedido un periodo transitorio de 15 años, finalizando en 2018, para la adecuación de las explotaciones ganaderas.

La Comunidad Valenciana (CV) se ha considerado como una región muy dinámica en cuanto al desarrollo urbanístico e industrial, el crecimiento demográfico y las actividades agrarias. El sector ganadero valenciano se caracteriza por una elevada industrialización, con explotaciones independientes del factor tierra, llegando a una situación de total separación entre ganadería y agricultura. La adaptación a las nuevas normativas ha supuesto una pérdida de competitividad en el sector ganadero.

En este contexto, la Administración Autonómica pretende establecer una serie de actuaciones sobre la ganadería con objeto de mejorar su competitividad y la cada vez más acusada baja productividad, en particular en las áreas vulnerables y de mayor dependencia del sector agrario. Se reclama el desarrollo urgente de medidas operativas, tecnológicas y económicas que lo dinamicen y permitan mantener las rentas de los habitantes $y$, al mismo tiempo, garantizar un desarrollo sostenible, cumpliendo así con las nuevas normativas [ORDEN 7/2010, Ministerio de Agricultura, Pesca y Alimentación (DOCV, 2010)].

El incremento competitivo de las explotaciones tan sólo será posible si se mejora la efi- ciencia en la gestión. En este sentido, se han realizado estudios sobre la eficiencia de granjas tanto desde el punto de vista económico como medioambiental, utilizando inputs y outputs relacionados con su función de producción o con la generación de externalidades negativas en el medioambiente (Piot-Lepetit y Vermersch, 1998; Lansink y Reinhard 2004; Asmild y Hougaard 2006; Yang et al., 2008; Yang 2009). Todos ellos concluyen que los propietarios tienen un comportamiento secuencial en la toma de decisiones, siendo la mejora de la eficiencia económica su objetivo principal, considerando adicional los avances medioambientales. De ahí que se califique como primordial la estimación de la eficiencia económica para la formulación de políticas.

La preocupación por el análisis de la situación económica de la ganadería ha despertado el interés en muchos países y regiones. En este panorama, los estudios de eficiencia son cada vez más numerosos en las distintas especies y áreas de interés ganadero, como es el caso del vacuno de carne (Iraizoz y Atance, 2004; Castillo, 2006), leche (González et al., 1996; Pardo et al., 2001; Ribas et al., 2006), aves (Chirinos y Urdaneta, 2007), así como en dehesas, comparando sistemas de producción intensivos con extensivos (Gaspar et al., 2007). Sin embargo, el porcino ha avivado un interés especial debido a las externalidades negativas generadas por sus subproductos contaminantes, causantes del desarrollo de nuevas normativas medioambientales. A nivel nacional, Murua y Albisu (1993) estudiaron la eficiencia en las granjas de porcino aragonesas, obteniendo que las más eficientes eran las de régimen integrado y aquellas donde se producía una renovación del capital humano. Por su parte, Láinez et al. (2002) describían las relaciones comerciales de las explotaciones porcinas en la CV a partir de la información suministrada por 202 granjas. Igualmente, esta inquietud se ha visto reflejada en investigaciones similares realizadas en otras áreas geográficas fuera de España [Francia 
(Piot-Lepetit y Vermersch, 1998), Hawái (Sharma et al. 1997 y 1999), Holanda (Lansink y Reinhard, 2004), Grecia (Galanopoulos et al., 2006), Dinamarca (Asmild y Hougaard, 2006) y Taiwán (Yang et al., 2008; Yang, 2009)].

Las perspectivas de futuro del sector porcino y avícola en la CV están limitadas por la situación de los mercados, tanto de productos finales como de materias primas (principalmente de cereales) y exigencias medioambientales. Además, existe poca vocación exportadora, son estructuralmente excedentarios, avocadas a abandonar la actividad todas aquellas explotaciones situadas en el límite de la eficiencia económica, es decir, en una posición marginal en términos económicos, incapaces de resolver su problemática ambiental (Láinez et al., 2002).

Siguiendo esta línea de investigación, el objetivo de este estudio consiste en analizar la situación económica de la industria ganadera de la CV, concretamente de porcino y aves. A partir de la estimación de una función de producción frontera (metodología DEA) con las explotaciones que alcancen mayores productividades, se medirá el nivel de eficiencia relativa, es decir, en relación a las explotaciones "mejores". Los resultados permitirán detectar la existencia de patrones que faciliten la corrección de errores con la finalidad de mejorar la competitividad del sector, ayudando a la definición de políticas adecuadas para cada escenario, y a la mejora del crecimiento en capacidad y ahorro de recursos. La aportación novedosa de este estudio reside en estudiar, desde un ámbito económico, dos sectores ganaderos con amplia aportación al PIB de la CV, construyendo para ello inputs y outputs no identificables directamente en el Censo Agrario. Ha sido necesaria la transformación de determinadas variables procedentes de varias fuentes estadísticas [Instituto Nacional de Estadística (INE)-Censo agrario (INE, 2012) y RECAN-Red Contable Agraria Nacional del Ministerio de Agricultura, Alimenta- ción y Medio Ambiente (RECAN, 2009)], con objeto de obtener una base de datos compuesta por una diversidad de variables económicas representativas del colectivo. Además, la muestra agrupa la casi totalidad de las granjas existentes en la zona analizada. La hipótesis de partida fue que el comportamiento de las políticas sectoriales no ha tenido la misma incidencia en la situación económica de las dos especies analizadas.

\section{Material y métodos}

\section{Caracterización de las explotaciones ganaderas en la Comunidad Valenciana}

El sector ganadero de la CV tiene una clara orientación hacia aquellas actividades susceptibles de producción intensiva, coherente con la existencia de grandes centros urbanos de consumo, elevada densidad demográfica y escasez de pastos naturales. De ahí que la producción porcina y avícola alcance el $60 \%$ del total obtenido por la ganadería. A diferencia de la agricultura, este subsector está más profesionalizado, tiene una dimensión económica por explotación mayor y una estructura de empleo más familiar.

Actualmente en la CV existen un total de 3.072 explotaciones, distribuyéndose en 21 especies diferentes, destacando el ovino $(21,03 \%)$, porcino de engorde $(18,8 \%)$ y pollos de engorde (13,74\%). Según la información que se puede extraer del último censo agrario publicado en 2009, el sector porcino está muy desproporcionado en cuanto a la cantidad de explotaciones que se dedican al engorde (516) frente a las de cría (93), lo que supone una mayor dependencia de otras zonas especializadas en la cría de cerdos de reducida edad (Tabla 1).

Las explotaciones de porcino se sitúan, generalmente, fuera de las comarcas del litoral, encontrándose más desarrolladas en zonas 
Tabla 1. Las clases de dimensiones económicas (DE) de las explotaciones y sus límites según la Producción total estándar. Número de granjas de porcino y aves en cada DE

Table 1. Economic dimensión (ED) clases and their limits according the standard total production. Number of pig and poultry farms in each ED

\begin{tabular}{|c|c|c|c|c|c|}
\hline \multirow{2}{*}{$\begin{array}{l}\text { Dimensión } \\
\text { Económica } \\
\text { Clase }\end{array}$} & \multirow{2}{*}{$\begin{array}{l}\text { Producción estándar total (PET) } \\
\text { Límites en } €\end{array}$} & \multicolumn{2}{|c|}{ Porcino } & \multicolumn{2}{|c|}{ Aves } \\
\hline & & No. & $\%$ & No. & $\%$ \\
\hline 1 & menos de 2.000 & 0 & 0 & 2 & 0,47 \\
\hline 2 & de 2.000 a menos de 4.000 & 0 & 0 & 1 & 0,24 \\
\hline 3 & de 4.000 a menos de 8.000 & 1 & 0,19 & 1 & 0,24 \\
\hline 4 & de 8.000 a menos de 15.000 & 1 & 0,19 & 1 & 0,24 \\
\hline 5 & de 15.000 a menos de 25.000 & 1 & 0,19 & 0 & 0,00 \\
\hline 6 & de 25.000 a menos de 50.000 & 5 & 0,97 & 3 & 0,71 \\
\hline 7 & de 50.000 a menos de 100.000 & 30 & 5,81 & 11 & 2,61 \\
\hline 8 & de 100.000 a menos de 250.000 & 232 & 45,54 & 128 & 30,33 \\
\hline 9 & de 250.000 a menos de 500.000 & 179 & 34,88 & 171 & 40,52 \\
\hline 10 & de 500.000 a menos de 750.000 & 39 & 7,56 & 52 & 12,32 \\
\hline 11 & de 750.000 a menos de 1.000 .000 & 11 & 2,13 & 31 & 7,35 \\
\hline 12 & de 1.000 .000 a menos de 1.500 .000 & 7 & 1,36 & 12 & 2,84 \\
\hline 13 & de 1.500 .000 a menos de 3.000 .000 & 4 & 0,78 & 6 & 1,42 \\
\hline \multirow[t]{2}{*}{14} & de 3.000 .000 o más & 2 & 0,39 & 3 & 0,71 \\
\hline & Total & 516 & 100 & 422 & 100 \\
\hline
\end{tabular}

Las celdas sombreadas resaltan las dimensiones económicas más representativas, es decir, aquellas que cuentan con mayor número de explotaciones de cada especie.

Fuente: Cuentas contables de la RECAN (2009), y elaboración propia a partir del Censo (2009) (INE, 2012) Se han eliminado 23 explotaciones de porcino por ser consideradas como outliers.

desfavorables económicamente, donde la ganadería desempeña un papel fundamental en el mantenimiento de la población rural. Ahora bien, aunque aportan incrementos significativos en las rentas familiares, también ocasionan una concentración de purines altamente contaminantes para suelos y aguas superficiales (Tamminga, 2003), y son una fuente emisora de amonio y gases efecto in- vernadero, tales como el metano y óxido nitroso (Beccaccia et al., 2015). Se trata de una actividad caracterizada por estar altamente vertebrada, con una integración vertical y muy industrializada, estando vinculada a la economía familiar del medio rural.

Por el contrario, las granjas de aves originariamente podían encontrarse a lo largo de toda la Comunidad, llegando en ocasiones a 
ser clave para asegurar la renta de la población y su establecimiento en determinadas áreas rurales. Sin embargo, en las últimas décadas, la presión del sector servicios y el desarrollo urbanístico en la zona costera, han desplazado las áreas avícolas a comarcas interiores, suponiendo un aumento de los costes de comercialización, y por tanto, afectando a su rentabilidad.

La mayoría de explotaciones de porcino de engorde ubicadas en tierras valencianas pertenecen a las Dimensiones Económicas (DE) 8, 9 y 10, es decir, tienen una Producción Estándar Total (PET) comprendida entre $100.000 \mathrm{y}$ 750.000 euros (Tabla 1). También, las estadísticas indican que el $86 \%$ de las granjas de aves de engorde pertenecen a las clases 8, 9, $10 \mathrm{y}$ 11. Ambas especies se caracterizan por una alta concentración en DE elevadas.
La información del Censo Agrario ha permitido caracterizar a nivel provincial las explotaciones de la CV (Tabla 2), atendiendo a su ubicación territorial, al número de explotaciones y a su capacidad productiva en términos de Unidad de Ganado Mayor (UGM).

Geográficamente existe una concentración de granjas de porcino y aves en las tierras de Castellón y Valencia $(59,3 \%$ y $65,9 \%$ en Castellón y $39,1 \%$ y $29,4 \%$ en Valencia, respectivamente) frente a las encontradas en Alicante (Tabla 2). La revisión de los censos agrarios anteriores confirma que esta realidad ha permanecido constante en el tiempo, de ahí que este escenario no responde a ninguna circunstancia coyuntural. Además, la situación minoritaria de Alicante se repite cuando se analiza su capacidad productiva (UGM), en porcino tan solo representa el $2,1 \%$ y en aves el $3,1 \%$.

Tabla 2. Distribución de las granjas por tipos y provincias Table 2. Farms distribution by product orientation and provinces

\begin{tabular}{|c|c|c|c|c|c|c|c|c|}
\hline & \multicolumn{4}{|c|}{ Porcino } & \multicolumn{4}{|c|}{ Aves } \\
\hline & $\mathrm{N}^{\circ 1}$ & $\%$ & $\mathrm{UGM}^{2}$ & $\%$ & $\mathrm{~N}^{01}$ & $\%$ & $\mathrm{UGM}^{2}$ & $\%$ \\
\hline Alicante & 8 & 1,6 & $4.109,9$ & 2,1 & 20 & 4,7 & $3.696,5$ & 3,1 \\
\hline Castellon & 306 & 59,3 & $117.144,1$ & 60,4 & 278 & 65,9 & $74.974,9$ & 63,2 \\
\hline Valencia & 202 & 39,1 & $72.707,1$ & 37,5 & 124 & 29,4 & $39.871,0$ & 33,6 \\
\hline$\overline{\mathrm{CV}^{3}}$ & 516 & 100 & $193.961,1$ & 100 & 422 & 100 & $118.542,4$ & 100 \\
\hline
\end{tabular}

${ }^{1}$ Número de explotaciones, ${ }^{2}$ Unidades de Ganado Mayor, ${ }^{3}$ Comunidad Valenciana.

Fuente: Elaboración propia a partir del Censo (2009).

Por otra parte, las granjas de las dimensiones económicas más importantes se pueden caracterizar por su ubicación, situación jurídica y capacitación agrícola (Tabla 3 ).

En las DE analizadas, Castellón aglutina más de la mitad de las granjas de porcino y aves de engorde, seguido de Valencia y siendo muy residual en Alicante. Al igual que ocurría en el total de la muestra, se trata de explotaciones gestionadas por una persona física, normalmente el propietario, con gran experiencia agraria y, en el caso del porcino, con menores estudios universitarios. Las granjas avícolas se caracterizan por la mayor demanda de personal cualificado, en donde 
Tabla 3. Distribución de explotaciones de porcino y aves de cada DE más representativa Table 3. Distribution of pig and poultry farms within the most representative economic dimensions (ED)

\begin{tabular}{|c|c|c|c|c|c|c|c|c|}
\hline & & \multicolumn{3}{|c|}{ Porcino (\%) } & \multicolumn{4}{|c|}{ Aves (\%) } \\
\hline & & ED8 & ED9 & ED10 & ED8 & ED9 & ED10 & ED11 \\
\hline \multirow[t]{3}{*}{ Provincia } & Alicante & 0,9 & 1,7 & 2,63 & 2,63 & 5,16 & 0 & 0 \\
\hline & Castellon & 59,9 & 59,2 & 75,44 & 75,44 & 63,23 & 70,59 & 60,00 \\
\hline & Valencia & 39,2 & 39,1 & 21,93 & 21,93 & 31,61 & 29,41 & 40,00 \\
\hline \multirow[t]{4}{*}{ Condición jurídica } & Persona física & 92,7 & 81,0 & 93,86 & 93,86 & 78,06 & 84,31 & 66,67 \\
\hline & Sociedad Mercantil & 1,7 & 9,5 & 0,88 & 0,88 & 5,80 & 5,88 & 16,67 \\
\hline & Sociedad cooperativa & 0,4 & 2,8 & 0 & 0 & 1,29 & 1,96 & 0 \\
\hline & Otras condiciones jurídicas & 5,2 & 6,7 & 5,26 & 5,26 & 14,84 & 7,84 & 16,67 \\
\hline \multirow[t]{4}{*}{ Formación agrícola } & Experiencia agraria & 80,2 & 71,5 & 73,68 & 73,68 & 63,87 & 72,55 & 53,33 \\
\hline & Cursos agrarios & 3,9 & 2,8 & 1,75 & 1,75 & 5,16 & 5,88 & 0 \\
\hline & Estudios profesionales agrarios & 0 & 0 & 0,82 & 0,82 & 0,65 & 0 & 0 \\
\hline & Estudios universitarios agrarios & 15,9 & 25,7 & 23,63 & 23,63 & 30,32 & 21,52 & 46,67 \\
\hline
\end{tabular}

Fuente: Elaboración propia.

se comparte la forma jurídica de persona física con otras formas de organización. Ello se debe a que estas explotaciones están muy tecnificadas y requieren controles de las explotaciones más específicos.

\section{Análisis Envolvente de Datos}

El Análisis Envolvente de Datos (DEA) es una técnica no paramétrica que permite medir la eficiencia relativa de unidades homogéneas. Este método es uno de los más utilizados en presencia de múltiples inputs y outputs para determinar cuáles son las mejores observaciones a través de la comparación de cada una con todas las combinaciones lineales posibles de las variables del resto de la muestra, pudiendo definir posteriormente con ellas una frontera de producción empírica. Así, la eficiencia de cada unidad analizada se mide como la distancia a la frontera.
Siguiendo al trabajo pionero de Farrell (1957), el modelo DEA fue desarrollado por Charnes et al. (1978) con el objetivo de encontrar el conjunto óptimo de pesos que maximice la eficiencia relativa $\left(h_{0}\right)$ de la empresa evaluada, definida ésta como el cociente entre la suma ponderada de outputs e inputs, sujeto a la restricción de que ninguna otra empresa puede tener una puntuación de eficiencia mayor que la unidad usando los mismos pesos. En concreto, el problema de programación lineal original basado en la orientación input y con rendimientos constantes de escala es el siguiente:

$$
\begin{gathered}
\operatorname{Max}_{u, v} h_{0}=\frac{\sum_{r=1}^{s} u_{r} * y_{r 0}}{\sum_{i=1}^{m} v_{i} * x_{i 0}} \\
\text { s.a. }=\frac{\sum_{r=1}^{s} u_{r} * y_{r j}}{\sum_{i=1}^{m} v_{i}{ }^{*} x_{i j}} \leq 1 \\
u_{r}, v_{i} \geq 0
\end{gathered}
$$


donde:

$\mathrm{x}_{\mathrm{ij}}$ : cantidades de input $\mathrm{i}(\mathrm{i}=1,2, \ldots . ., \mathrm{m})$ consumidos por la j-ésima explotación ganadera.

$\mathrm{x}_{\mathrm{i} 0}$ : cantidades de input i consumidos por la explotación evaluada.

$\mathrm{y}_{\mathrm{rj}}$ : cantidades de output $\mathrm{r}(\mathrm{r}=1,2, \ldots, \mathrm{s})$ producidos por la j-ésima explotación ganadera.

$\mathrm{y}_{\mathrm{r} 0}$ : cantidades de output $\mathrm{r}$ producidos por la explotación ganadera evaluada.

$\mathrm{u}_{\mathrm{r}}$ : ponderación de los outputs.

$\mathrm{v}_{\mathrm{i}}$ : ponderación de los inputs.

Sin embargo, en el presente artículo se ha resuelto el problema dual correspondiente al modelo linealizado porque de esta forma el número de variables es mayor que el de las restricciones. Esto supone como limitación que no se conocen directamente las ponderaciones de los inputs y outputs considerados.

En la aplicación de la técnica DEA la elección en la orientación depende de la habilidad de cada observación para controlar la cantidad de outputs o inputs. Dado que en España no existen topes de producción ganadera se ha empleado el modelo output, indicando cuánto podría obtenerse de output con el mismo nivel de inputs en ausencia de ineficiencias ${ }^{1}$.

La medida de eficiencia está comprendida entre los valores 0 y 1 , siendo su interpretación la siguiente:

- $\mathrm{Si}_{0}=1$, la explotación es eficiente con relación a las otras y, por tanto, estará situada en la frontera de producción.

- Si $h_{0}<1$, otra explotación es más eficiente que la analizada, siendo identificada como aquella que deberá mejorar su gestión para conseguir un mejor funcionamiento.

Al igual que ocurre en otros trabajos, en el ámbito de la ganadería el concepto de eficiencia que se pretende cuantificar no es estrictamente el de eficiencia técnica (Castillo, 2006; Ribas et al., 2006; Chirinos y Urdaneta, 2007), debido a que el gasto corriente es un input expresado en términos monetarios. Por tanto, será un tipo de eficiencia híbrida, cercano al concepto de eficiencia técnica pero sin llegar a ser asignativa ya que no se incluye el precio relativo de los inputs, cuya dificultad de obtención es considerable en este ámbito.

La aplicación de la metodología DEA precisa de la definición de los inputs y outputs que constituirán el modelo. Además, ha sido necesario el tratamiento de las explotaciones agrarias como una "industria" capaz de transformar unos recursos en producción final (output).

Se ha tomado como único output la Producción Estándar Total (PET) entendida como el valor monetario bruto (euros) del porcino y de las aves a precio de salida de la explotación. Mientras que las variables que definen los inputs cubren todos los aspectos inherentes a la explotación porcina y avícola. Concretamente son las siguientes:

- Superficie Agraria Útil (SAU): Superficie en Ha. de tierras para pastos permanentes y tierras labradas.

- Unidad de Trabajo-Año totales (UTAT): Horas de trabajo en las explotaciones expresadas en número de jornadas. Una UTA equivale al trabajo que realiza una persona a tiempo completo a lo largo de un año, es decir, 1.826 horas/año.

1. Otros trabajos también han utilizado este enfoque (Arzubi y Berbel, 2001; Castillo, 2006; Chirinos y Urdaneta, 2007). El programa utilizado para calcular los valores de eficiencia ha sido DEAP versión 2.1 (Coelli, 1996). 
- Unidad de Ganado Mayor (UGM): Equivale a una cabeza de ganado de referencia. Se calcula multiplicando el número de cabezas de ganado por un factor de ponderación, dependiendo de la raza y tipo de animal. Lo más común es que la especie de referencia sea el bovino adulto. En este trabajo se han seguido las recomendaciones de EUROSTAT, considerando que para el porcino de engorde el factor de ponderación es 0,3 y 0,03 para las aves de engorde.

- Costes Específicos de las Explotaciones Ganaderas: Costes de alimentación y otros costes específicos de ganadería (Gastos veterinarios y reproducción, los incurridos en la preparación para el mercado, almacenamiento, así como comercialización de productos ganaderos).

- Costes Generales de Explotación: Gastos ligados con la actividad productiva, diferentes de los específicos, definidos por:

- Costes corrientes: Costes de adquisición de equipos pequeños.

- Costes de mantenimiento y reparaciones: Teniendo en cuenta que las reparaciones de mayor cuantía que aumentan el valor de los edificios y maquinaria, están consideradas como inversiones.

- Costes en energía: Consumo de carburantes y lubricantes para motores, así como electricidad y carburantes para calefacción.

- Otros costes directos: Consumo de agua, seguros (excepto los de edificios y accidentes de trabajo) y otros gastos generales de explotación (contabilidad, teléfono, etc.).

- Amortización: Depreciación de los activos fijos durante el ejercicio contable. Se determina a partir del valor de reposición al final del ejercicio de las plantaciones de cultivos permanentes, edificios de la explotación, mejoras de la tierra, maquinaria y equipos.
- Costes de factores externos: Incluye los salarios y cargas sociales, arrendamientos e intereses pagados.

La base de datos ha sido construida a partir de los microdatos facilitados por el INE-Censo Agrario de 2009 (INE, 2012) y la RECAN-Red Contable Agraria Nacional de 2009 (RECAN, 2009). El Censo es elaborado periódicamente por el Instituto Nacional de Estadística (INE) mediante un proceso de encuestas sobre métodos de producción en las explotaciones agrícolas. Dada la complejidad de su elaboración, se publica siempre con cierto retraso, de ahí que la última información disponible sea la correspondiente a 2009.

Por su parte, la RECAN procede del Ministerio de Agricultura, Alimentación y Medio Ambiente (MAGRAMA), la cual cada año, mediante un sistema de muestreo, realiza encuestas con el objetivo de recoger datos contables de las granjas y hacer un seguimiento de los resultados de explotación de las actividades agrarias. Evidentemente, no es posible abarcar todas las granjas, por ello dedican gran esfuerzo en asegurarse que la muestra analizada sea representativa del universo global, teniendo en cuenta la región, el tamaño y el tipo de explotación. Se trata de una herramienta eficaz para valorar el impacto de las medidas adoptadas en el marco de los tratados de la Política Agraria Común, ya que contiene variables que pueden ser consideradas representativas de las gestión de las explotaciones (Dono et al., 2013). Se han utilizado los principios contables de la European Commission (2012) - Farm Accountancy Data Network (FADN), con el fin de ser comparables con el resto de países de la Unión Europea, y proporcionar información armonizada para toda Europa.

Las variables PET, SAU, UGM y UTAT se han obtenido a partir de los microdatos del Censo Agrario para cada explotación, el resto de inputs proceden de la RECAN del MAGRAMA. 
Previa consulta específica, el MAGRAMA elaboró una base de datos anonimizada con los valores de las encuestas realizadas por la RECAN de las granjas de porcino y aves de engorde de la CV, después de comprobar que el número de encuestas era representativa del total de explotaciones existente en la CV. En esta base de datos se agruparon los resultados según la DE en cada una de las especies ganaderas solicitadas. Las variables que incluían esta base de datos eran datos en unidades físicas (UGM, unidades de trabajo, etc.) y monetarias (costes). Con esta información se determinaron los costes por UGM mediante la siguiente expresión:

$$
\text { CosteUGM } M_{i j}=\frac{\text { coste }_{i j}}{U G M_{j}}
$$

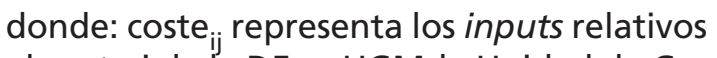
al gasto i de la $D E_{j}$; y UGM $\mathrm{UG}_{\mathrm{j}}$ la Unidad de Ganado Mayor de la $\mathrm{DE}_{\mathrm{j}}$.

Por otra parte, la información que proporciona el Censo Agrario (PET) permite clasificar las explotaciones ganaderas según la DE establecidas por la RECAN (Tabla 1), facilitándose de este modo la extrapolación de los resultados de la ecuación 2 al conocer el output de cada explotación. Finalmente, realizada la asignación por tamaño económico, y con objeto de obtener cada una de las variables por explotación, los Coste UGM han sido nuevamente multiplicados por su UGM correspondiente. Obteniendo la base de datos definitiva que contiene para todas las explotaciones todos los inputs considerados. Finalmente se eliminaron los datos outliers $^{2}$, obteniendo una base de datos con 434 explotaciones de porcino y 350 de aves.

Con objeto de acotar con más precisión el patrón que define las explotaciones eficien- tes será necesario analizar las variables ligadas a ella, es decir, los inputs de las granjas más eficientes, facilitando la concreción de su perfil. Para ello se utilizará el test no paramétrico de Kruskal-Wallis para categorizar los inputs en dos rangos (con niveles de eficiencia mayor o menor al $70 \%$ ), y facilitar la valoración de las posibles diferencias existentes en las variables de ambos grupos.

\section{Resultados}

Los principales estadísticos de las variables que componen el modelo utilizado en el análisis empírico permiten identificar cual es el tamaño medio de la muestra utilizada así como su dispersión y sus valores extremos definiendo las características del colectivo a estudiar (Tabla 4).

Se aprecia una gran dispersión, de manera que engloba tanto pequeñas granjas con producción limitada, como explotaciones cuyo output casi alcanza el millón de euros en el caso de las aves y los 750 mil euros en el porcino, de ahí la elevada desviación típica de todas las variables. Esta es una de las características fundamentales de las explotaciones de la CV. Las explotaciones de porcino obtienen una producción media superior a 250 mil euros, en promedio disponen de 2,03 Ha. de SAU, emplean 1,04 UTAT y su cabaña ronda las 273 UGM. Mientras que las aves obtienen una producción media superior a 385 mil euros, mayoritariamente disponen de 11 Ha. de SAU, contratan 1,35 UTAT y su cabaña ronda las 240 UGM. Por tanto, es posible concluir que las explotaciones avícolas, son más intensivas ya que, con menos UGM, requieren más trabajo, elevados costes de producción y obtienen una mayor producción.

2. Se identifican como aquellos datos que no pertenecen al intervalo formado por la media +/- tres veces la desviación típica en cada una de las variables. 
Tabla 4. Principales estadísticos de los outputs e inputs en porcino y aves Table 4. Main statistics for outputs and inputs in pig and poultry

\begin{tabular}{|c|c|c|c|c|c|}
\hline & Variables & Media & Máximo & Mínimo & Deviación Típica \\
\hline \multicolumn{6}{|c|}{ PORCINO } \\
\hline Output & $\operatorname{PET}(€)$ & $252.632,47$ & 745.155 .63 & $102.922,35$ & $141.927,91$ \\
\hline \multirow[t]{10}{*}{ Inputs } & SAU (Ha) & 2,03 & 91,18 & 0,01 & 12,81 \\
\hline & UTAT & 1,04 & 3,55 & 0,01 & 0,54 \\
\hline & UGM & 273,22 & $1.035,00$ & 13,50 & 209,39 \\
\hline & Coste Específicos $(€)$ & $138.964,35$ & $487.654,64$ & $6.539,54$ & $108.718,76$ \\
\hline & Costes Corrientes $(€)$ & 11,57 & $1.816,95$ & 0,01 & 614,42 \\
\hline & Costes Mantenimiento $(€)$ & $3.890,37$ & $9.105,16$ & 225,06 & $2.057,36$ \\
\hline & Energía $(€)$ & $2.496,29$ & $7.407,26$ & 123,39 & $1.791,76$ \\
\hline & Otros, Costes Generales $(€)$ & $2.574,48$ & $6.663,10$ & 145,21 & $1.444,61$ \\
\hline & Amortización $(€)$ & $5.491,12$ & $12.348,68$ & 330,19 & $2.756,37$ \\
\hline & Costes Externos $(€)$ & 498,79 & $4.840,80$ & 9,72 & $1.570,08$ \\
\hline \multicolumn{6}{|c|}{ AVES } \\
\hline Output & $\operatorname{PET}(€)$ & $385.669,35$ & $991.200,00$ & $101.359,83$ & $222.050,44$ \\
\hline \multirow[t]{10}{*}{ Inputs } & SAU (Ha) & 11,06 & 335,63 & 0,001 & 30,00 \\
\hline & UTAT & 1,35 & 8,00 & 0,04 & 0,81 \\
\hline & UGM & 239,65 & 945,00 & 56,00 & 161,55 \\
\hline & Coste Específicos $(€)$ & $199.713,11$ & $728.775,73$ & $67.005,27$ & $102.973,11$ \\
\hline & Costes Corrientes $(€)$ & 203,48 & 870,42 & 0,06 & 196,42 \\
\hline & Costes Mantenimiento $(€)$ & $16.372,31$ & $78.387,19$ & $1.178,32$ & $11.893,72$ \\
\hline & Energía $(€)$ & $8.436,47$ & $25.056,87$ & $2.545,69$ & $4.366,66$ \\
\hline & Otros, Costes Generales $(€)$ & $3.599,78$ & $8.442,84$ & $1.455,37$ & $1.343,69$ \\
\hline & Amortización $(€)$ & $6.988,73$ & $18.275,79$ & $2.785,33$ & $2.851,56$ \\
\hline & Costes Externos $(€)$ & $2.328,44$ & $6.697,66$ & 448,54 & $1.437,65$ \\
\hline
\end{tabular}

PET: Producción estándar Total; SAU: Superficie Agraria Útil; UTAT: Unidad de Trabajo-Año Totales; UGM: Unidad de Ganado Mayor.

Fuente: Elaboración propia. 
La aplicación del modelo DEA ha permitido obtener los resultados de eficiencia de cada explotación, los cuales representan un indicador de la gestión realizada en un momento determinado. El nivel de eficiencia de una granja es relativo, puesto que viene condicionado por las otras unidades de la muestra con quien se compara. Todas las observaciones representan, dentro de su categoría, un colectivo homogéneo en cuanto a su proceso productivo. En este caso se trata de medir la eficiencia de las granjas de porcino y aves sobre una muestra de 434 y 350 explotaciones, respectivamente (Tabla 5).

Tabla 5. Resultados de eficiencia en porcino y aves en cada provincia y en las dimensiones económicas (DE) con mayor concentración de granjas

Table 5. Efficiency results for pig and poultry, across provinces, and within the most representative economic dimensions (ED)

\begin{tabular}{lccccc}
\hline & $N^{\circ}$ granjas & $\begin{array}{c}N^{\circ} \text { granjas } \\
\text { eficientes }\end{array}$ & $\begin{array}{c}\text { Eficiencia } \\
\text { media }\end{array}$ & $\begin{array}{c}\text { Eficiencia } \\
\text { máxima }\end{array}$ & $\begin{array}{c}\text { Eficiencia } \\
\text { mínima }\end{array}$ \\
\hline AL & 2 & \multicolumn{2}{c}{ PORCINO } & & \\
CS & 253 & 14 & 0,68 & 0,73 & 0,63 \\
VL & 179 & 26 & 0,68 & 1,00 & 0,40 \\
DE8 & 226 & 24 & 0,70 & 1,00 & 0,39 \\
DE9 & 170 & 6 & 0,59 & 1,00 & 0,42 \\
DE10 & 38 & 10 & 0,90 & 1,00 & 0,39 \\
\hline & 11 & 0 & 1,00 & 0,90 \\
\hline AL & 238 & 40 & 0,90 & 0,99 & 0,82 \\
CS & 101 & 34 & 0,88 & 1,00 & 0,53 \\
VL & 114 & 17 & 0,92 & 1,00 & 0,58 \\
DE8 & 155 & 10 & 0,93 & 1,00 & 0,74 \\
DE9 & 51 & 20 & 0,84 & 1,00 & 0,53 \\
DE10 & 30 & 9 & 0,95 & 1,00 & 0,68 \\
DE11 & & 0,95 & 1,00 & 0,8 \\
\hline AL:Alicas & & & &
\end{tabular}

AL: Alicante; CS: Castellon; VL: Valencia.

Fuente: Elaboración propia.

En la CV el número de granjas situadas en la frontera es preocupantemente bajo en ambas especies, siendo más acusado en el porcino: tan sólo un 9,2\% de las granjas anali- zadas son completamente eficientes. De ahí que las empresas ganaderas deberían mejorar la gestión de sus inputs con objeto de incrementar su producción. El caso extremo se 
encuentra en Alicante donde ninguna explotación es eficiente, aunque en las aves sus niveles máximos llegan a estar próximos a uno.

Respecto a los valores medios se aprecian diferencias significativas entre las dos especies analizadas. Mientras que el sector de las aves está, por término medio, bastante próximo a la frontera $(0,9)$, el porcino todavía tiene un amplio recorrido de mejora $(0,68)$, es decir, las aves con el mismo volumen de inputs podrían incrementar su producción un $10 \%$, sin embargo en el caso de los cerdos se podría aumentar $32 \%$. En términos absolutos, son eficientes 40 granjas de porcino y 74 de aves, además estas últimas están más próximas a la frontera y su eficiencia mínima es muy superior al porcino $(0,53$ frente a 0,39$)$.
Si se comparan los resultados de eficiencia distinguiendo las clases de DE que recogen el mayor número de granjas de porcino y aves, se pueden identificar las diferencias entre los grupos (Tabla 5). Estos resultados muestran que tanto en el porcino como en las aves son las granjas de tamaño medio (DE9) las que tienen que esforzarse más en gestionar mejor sus inputs, tal y como lo hacen las de mayor dimensión (DE10 y, en aves, DE11).

Por otra parte, las características intrínsecas de las explotaciones eficientes, según su condición jurídica y la formación del personal también permiten identificar el patrón de comportamiento de las mismas (Tabla 6).

La mayoría de las granjas eficientes siguen el mismo patrón que el total de la muestra, con la

Tabla 6. Caracterización jurídica y cualificación de los trabajadores de las granjas (\%) Table 6. Legal status and farmers' qualification (\%)

\begin{tabular}{|c|c|c|c|c|}
\hline \multirow[b]{2}{*}{ Condición jurídica } & \multicolumn{2}{|c|}{ Eficientes } & \multicolumn{2}{|c|}{ No eficientes } \\
\hline & Porcino & Aves & Porcino & Aves \\
\hline Persona física & 82,50 & 78,38 & 87,82 & 84,42 \\
\hline Sociedad Mercantil & 7,50 & 5,41 & 4,82 & 5,07 \\
\hline Sociedad cooperativa & 0 & 1,35 & 1,52 & 0,72 \\
\hline Otras condiciones jurídicas & 10,00 & 14,86 & 5,84 & 9,78 \\
\hline Formación del personal & Porcino & Aves & Porcino & Aves \\
\hline Experiencia agraria & 72,50 & 70,27 & 76,90 & 66,67 \\
\hline Cursos agrarios & 10,00 & 2,7 & 3,30 & 3,99 \\
\hline Estudios profesionales agrarios & 0 & 1,35 & 0 & 0,36 \\
\hline Estudios universitarios agrarios & 17,5 & 25,68 & 19,80 & 28,99 \\
\hline
\end{tabular}

Fuente: Elaboración propia.

diferencia que aumenta el porcentaje de granjas constituidas como una sociedad mercantil y, sobretodo, como otras formas jurídicas. Además, muchas de éstas han sido constituidas a través de una persona física, sin cualificación, pero aumenta el número de granjas en las que el jefe de explotación realiza cursos agrarios específicos, y por tanto, aunque se han basado en su experiencia para dirigir la producción, realizan cursos de formación para 
mejorar su gestión. En el sector avícola, la condición jurídica del ganadero es mayoritariamente persona física, sin embargo existe un volumen mayor de sociedades mercantiles y otras condiciones jurídicas (comunidad de bienes, sociedad civil, etc.). Su alto nivel de ex- periencia se une a un mayor porcentaje de personal con formación agraria.

La utilización del test no paramétrico de Kruskal-Wallis (Tabla 7) muestra las diferencias existentes en las variables de ambos grupos

Tabla 7. Resultado del Test Kruskal-Wallis sobre las granjas de porcino y aves Table 7. Kruskal-Wallis test results applied to pig and poultry farms

\begin{tabular}{|c|c|c|c|c|c|}
\hline & Todas & Efic. $<70 \%$ & Efic. $>70 \%$ & Estadístico & $\mathrm{p}$-value \\
\hline \multicolumn{6}{|c|}{ PORCINO } \\
\hline $\mathrm{N}^{\circ}$ granjas & 434 & 225 & 209 & & \\
\hline SAU & 9,6 & 9,8 & 9,3 & 4,195 & 0,041 \\
\hline UTAT & 1,2 & 1,2 & 1,2 & 0,059 & 0,808 \\
\hline UGM & 347,9 & 347,9 & 348,0 & 1,087 & 0,297 \\
\hline Costes Específicos & $179.377,2$ & $186.922,4$ & $171.254,5$ & 1,493 & 0,221 \\
\hline Costes Corrientes & 520,9 & 729,1 & 296,8 & 9,864 & 0,001 \\
\hline Costes Mantenimiento & $4.656,8$ & $4.837,0$ & $4.462,8$ & 0,926 & 0,335 \\
\hline Energía & $3.167,3$ & $3.621,0$ & $2.678,8$ & 17,185 & 0,000 \\
\hline Otros Costes Generales & $3.105,4$ & $3.159,1$ & $3.047,6$ & 0,124 & 0,724 \\
\hline Amortizaciones & $6.518,3$ & $6.591,3$ & $6.439,8$ & 0,630 & 0,427 \\
\hline Costes Externos & $1.367,9$ & $1.993,4$ & 694,5 & 12,703 & 0,000 \\
\hline \multicolumn{6}{|c|}{ AVES } \\
\hline $\mathrm{N}^{\circ}$ granjas & 350 & 21 & 329 & & \\
\hline SAU & 11,1 & 22,9 & 10,3 & 0,007 & 0,932 \\
\hline UTAT & 1,4 & 1,3 & 1,4 & 0,060 & 0,807 \\
\hline UGM & 239,7 & 330,3 & 233,9 & 17,447 & 0,000 \\
\hline Costes Específicos & $199.713,1$ & $304.324,3$ & $193.035,8$ & 31,883 & 0,000 \\
\hline Costes Corrientes & 203,5 & 430,4 & 189,0 & 35,046 & 0,000 \\
\hline Costes Mantenimiento & $16.372,3$ & $23.400,9$ & $15.923,7$ & 25,302 & 0,000 \\
\hline Energía & $8.436,5$ & $12.401,2$ & $8.183,4$ & 25,280 & 0,000 \\
\hline Otros Costes Generales & $3.599,8$ & $6.407,9$ & $3.420,5$ & 48,340 & 0,000 \\
\hline Amortizaciones & $6.988,7$ & $7.286,7$ & $6.969,7$ & 1,463 & 0,227 \\
\hline Costes Externos & $2.328,4$ & $5.021,0$ & $2.156,6$ & 46,623 & 0,000 \\
\hline
\end{tabular}

SAU: Superficie Agraria Útil; UTAT: Unidad de Trabajo-Año Totales; UGM: Unidad de Ganado Mayor. Fuente: Elaboración propia. 
(granjas eficientes y no eficientes), donde la primera columna recoge la media de cada uno de los inputs sobre la muestra total, de forma que dicho valor servirá de referencia al promedio de aquellas granjas con niveles de eficiencia inferiores al $70 \%$ (columna 2 ) y a las que presentan niveles superiores (columna 3).

El estadístico chi-cuadrado del test de Kruskal-Wallis en las explotaciones de porcino ha resultado significativo para los costes corrientes, de energía y los externos, siendo estas variables las que permiten diferenciar la muestra según sus niveles de eficiencia. Las más eficientes se caracterizan por ser explotaciones que incurren en menores costes específicos, corrientes, de mantenimiento, energía y otros costes generales (Tabla 7). Por tanto, se trata de explotaciones productivas que saben gestionar bien sus recursos porque gastan menos y consiguen más producción.

En el sector avícola el estadístico chi-cuadrado de SAU, UTAT y Amortización no es significativo, estas variables no permiten diferenciar la muestra según sus niveles de eficiencia. Por tanto, la gestión de las explotaciones avícolas es muy diferente a las del porcino, en esta especie las más eficientes son aquellas que tienen un tamaño físico (UGM) próximo a la media, utilizan menos inputs, pero más trabajo (UTAT). Por tanto, las menos eficientes incurren en mayores costes, concretamente un $57 \%$, $56 \%$ y $30 \%$ más elevados en factores externos, corrientes y otros costes, respectivamente. Además, las más eficientes tienen una SAU cercana a la media pudiendo afirmar que han conseguido aprovechar las economías de escala para obtener producciones óptimas.

A partir de los resultados obtenidos se puede afirmar que los sectores porcino y avícola de la CV tienen un importante potencial de mejora, siendo necesario identificar las características de las explotaciones eficientes con objeto de realizar una implantación general de las mis- mas. El porcino ocupa un puesto preponderante en la ganadería de la Comunidad, pero necesita adaptarse a los cambios acontecidos para optimizar su productividad y, de este modo, mejorar sus niveles de competitividad.

\section{Discusión}

En la literatura se encuentran estudios con objetivos similares al planteado en zonas geográficas muy diversas, siendo más frecuentes en la última década debido a la problemática ambiental despertada por estas granjas. Sin embargo, la gran mayoría de ellos han centrado la discusión en una comparativa entre eficiencia económica y la resultante del cumplimiento de las actuales normativas medioambientales (Piot-Lepetit y Vermersch, 1998; Lansink y Reinhard, 2004; Asmild y Hougaard, 2006; Yang et al., 2008; Yang, 2009). Todos ellos concluyen que, previo a optimizar el potencial medioambiental, es necesario mejorar la eficiencia económica, debido a la implicación directa del propietario de la explotación.

Los avances medioambientales conllevan un beneficio público que afecta a la sociedad en su conjunto y difícilmente se traduce en mejoras en los resultados de explotación. Todas las modificaciones en la normativa medioambiental impuestas por las continuas políticas públicas están condicionando la productividad de las granjas. De ahí que la orientación de la investigación propuesta se centre en analizar la eficiencia económica, introduciendo un mayor detalle en los inputs con objeto de poder establecer el patrón de eficiencia en esta área.

En esta investigación, a diferencia de otras, las observaciones de la muestra engloban casi el total de explotaciones de las DE registradas en el Censo Agrario [en comparación con las 107 explotaciones del estudio realizado en Francia por Piot-Lepetit y Vermersch 
(1998), las 290 danesas en Asmild y Hougaard (2006), las 100 griegas en Galanopoulos et al. (2006), las 31 chinas en Yang et al. (2008) y Yang (2009) o las 69 en la dehesa de Extremadura (España) en el estudio de Gaspar et al. (2009)]. De este modo, se ha evitado obtener resultados sesgados, condicionados por comportamientos específicos localizados en áreas geográficas concretas y poco extensibles a otras zonas ganaderas.

Los resultados revelan que en la CV el porcino tiene un amplio recorrido de mejora en sus niveles de eficiencia, algo similar a lo obtenido en otros territorios, como Grecia o Taiwán. Concretamente, en el caso de Grecia, Galanopoulos et al. (2006) deducen que estas granjas podrían reducir sus inputs un $17 \%$ obteniendo el mismo nivel de output, y en Taiwán, Yang et al. (2008) concluyen que, en promedio, es posible aumentar el output un $52,8 \%$, manteniendo los mismos niveles de inputs. Sin embargo, no son comparables a las conclusiones de la investigación realizada en las dehesas de Extremadura (España) al tratarse de sistemas de producción extensivos y no intensivos como en la CV.

En estos estudios se enfatizan las diferencias de tamaño existentes. Normalmente en el porcino las granjas grandes son más eficientes, mostrando la necesidad de beneficiarse de los rendimientos crecientes a escala y abandonar las producciones reducidas que impiden aprovecharse de estas ventajas. Proponen a las Administraciones incentivos para aumentar la dimensión de las explotaciones, con objeto de facilitarles la adaptación a los cambios normativos del sector (reducciones purines, emisiones, etc.). Una eficiencia más elevada implica, en última instancia, mayores beneficios, cuestión clave para despertar la motivación de los propietarios por la adopción de nuevas técnicas.

El carácter minifundista del sector ganadero de la CV se refleja en el elevado número de explotaciones, y en el caso del porcino, de in- eficientes, como revela el estudio realizado. Al igual que en Grecia y Taiwán, resulta imprescindible incrementar el tamaño de las granjas de porcino con objeto de poder mejorar su rendimiento y facilitar el cumplimiento de la normativa ambiental. Por tanto, la solución pasa por reducir el número de explotaciones, y en ocasiones trasladarlas a zonas del interior donde hay menores conflictos por el uso del suelo. Ahora bien, debe tenerse presente que todo ello precisa de una compensación monetaria traducida en mayores costes, difícilmente asumibles por el propietario dada la situación actual.

\section{Conclusiones}

Las nuevas exigencias medioambientales han provocado una adaptación inmediata en el sector ganadero, afectando a sus estructuras productivas. La comparación del sector porcino y de aves desvela que en la CV la problemática medioambiental del porcino podría ser uno de los causantes de su deficiente gestión económica, teniendo en cuenta que ya han incorporado en los costes corrientes la carga económica de la adecuación de las balsas de purines para reducir los riesgos de contaminación de aguas subterráneas.

Se ha demostrado que el incremento de tamaño mejoraría sus resultados, sin embargo debe valorarse otros aspectos que hasta ahora se tienen descuidados. El perfil jurídico-administrativo de las granjas eficientes es muy frágil, se trata de personas físicas con experiencia en el sector pero sin nivel académico, incapaces de trasladar a la explotación las ventajas ofrecidas por las sinergias propias de las agrupaciones de ganaderos, características de las sociedades cooperativas. Además, se hace cada vez más necesario la formación de la mano de obra que permita la utilización de técnicas avanzadas, acordes con las necesidades de mayor productividad, mejorando así la ido- 
neidad del sector. Se requiere mano de obra motivada que apueste por una mejora en su formación para introducir avances en las explotaciones que se traduzcan en aumentos en la producción, tal y como se ha comenzado a realizar en el sector avícola.

Igualmente, sabiendo que las explotaciones de porcino se encuentran en zonas de interior bastante desfavorecidas económicamente, una mejora de su eficiencia podría atraer a población joven, facilitando un cambio generacional que ayude a ir profesionalizando el sector. Por el contrario, las explotaciones de aves están ubicadas en zonas más cercanas al litoral, donde los conflictos por el uso del suelo son mayores, y la población tiene bastante facilidad para cambiar de sector económico, lo que en parte explica sus mayores niveles de eficiencia.

Las granjas minifundistas características de la CV soportan un importante volumen de costes que difícilmente pueden trasladarse al precio de venta del producto sin perder competitividad. Las políticas agrarias dirigidas a este sector deberían potenciar el uso eficiente de los recursos disponibles, que en algún caso pasa por la agrupación de producciones en cooperativas, con el fin de aumentar el tamaño de las mismas. Además, una política de personal en cuanto a la organización del trabajo daría lugar a una mayor implicación del personal en los resultados productivos y económicos de la explotación.

\section{Agradecimientos}

Los autores reconocen la ayuda de MAGRAMA en la provisión de información de microdatos de la Red Nacional de Contabilidad Agraria (RECAN).

\section{Bibliografía}

Arzubi A, Berbel J (2001). Un análisis no paramétrico de eficiencia en explotaciones lecheras de Argentina. Revista Española de Estudios Agrosociales y Pesqueros 193: 119-142.

Asmild M., Hougaard JL (2006). Economic versus environmental improvement potentials of Danish pig farms. Agricultural Economics, 35: 171-181.

Beccaccia A, Ferrer $P$, Ibáñez MA, Estellés F, Rodríguez C, Moset V, de Blas C, Calvet S, García-Rebollar P (2015). Relationships among slurry characteristics and gaseous emissions at different types of commercial Spanish pig farms. Spanish Journal of Agricultural ResearchSpan, 13(1), e06-002: 1-15.

BOE (2003) Ley 6/2003, de 4 de marzo, de Ganadería de la Comunidad Valenciana. Boletín Oficial del Estado, núm. 81, de 4 de abril de 2003, pp. $13083-13116$

Castillo M (2006). Eficiencia técnica de la producción de vacuno de carne en la dehesa. Revista Española de estudios agrosociales y pesqueros, 212: 139-154.

Charnes A, Cooper WW, Rhodes E (1978). Measuring the efficiency of decision making units. European Journal of Operational Research, 2: 429-444.

Chirinos A, Urdaneta M (2007). Medición de la eficiencia en el sector avícola mediante índices de Malmquist. Agroalimentaria, 25: 95-107.

Coelli T (1996). A guide to deap Version 2.1: A data envelopment analysis (Computer) program, Cepa Working Paper 96/08. Centre for Efficiency and Productivity Analysis, University of New England, Australia, 49 p.

DOCV (2010) ORDEN 7/2010, de 10 de febrero, de la Conselleria de Agricultura, Pesca y Alimentación, por la que se aprueba el Código Valenciano de Buenas Prácticas Agrarias. Diario Oficial de la Comunidad Valenciana, num. 6212 de 23 de febrero de 2010, pp. 7239-7250.

Dono G, Giraldo L, Nazzaro E (2013). Contribution of the calving interval to dairy farm profitability: results of a cluster analysis of FADN data for a major milk production area in southern Italy. Spanish Journal of Agricultural, 11(4): 857-868. 
European Commission (2012). The Farm Accountancy Data Network. Definition of variables used in FADN standard results. Disponible en: http://fadn.pl/wp-content/uploads/2013/ 06/RICC-882-EN-rev9.1-Definitions-of-Variablesfinal.pdf (consultado: 20 de abril de 2017).

Farrell MJ (1957). The measurement of productive efficiency. Journal of the Royal Statistical Society, 120(A): 253-281.

Galanopoulos K, Aggelopoulos S, Kamenidou I, Mattas K (2006). Assessing the effects of managerial and production practices on the efficiency of commercial pig farming. Agricultural Systems, 88(2): 125-141.

Gaspar P, Escribano M, Mesías FJ, Pulido F, MartínezCarrasco F (2007). La eficiencia en explotaciones ganaderas de dehesa: una aproximación DEA al papel de la sostenibilidad y de las subvenciones comunitarias. Revista española de estudios agrosociales y pesqueros, (215-216): 185-209.

Gaspar P, Mesías FJ, Escribano M, Pulido F (2009). Assessing the technical efficiency of extensive livestock farming systems in Extremadura, Spain. Livestock Science, 121(1): 7-14.

González E, Álvarez A, Arias C (1996). Análisis no paramétrico de eficiencia en explotaciones lecheras. Investigación Agraria: Economía, 11(1): 173-190.

INE (2012) INEbase. Censo agrario 2009. Instituto Nacional de Estadística, Madrid.

Iráizoz B, Atance I (2004). Análisis de la eficiencia técnica en explotaciones ganaderas de vacuno de carne en España. Revista española de estudios agrosociales y pesqueros, 204: 67-94.

Láinez M, Balash S, Nuez T, Gargallo LM, Torres A (2002). Relaciones comerciales de las explotaciones porcinas de la Comunidad Valenciana. Investigación Agraria: Producción y Sanidad Animal, 17(1-2): 59-79.

Lansink AO, Reinhard S (2004). Investigating technical efficiency and potential technological change in Dutch pig farming. Agricultural Systems, 79(3): 353-367.
Murua JR, Albisu LM (1993). Eficiencia técnica en la producción porcina en Aragón. Investigación Agraria: Economía, 8(2): 239-251.

Pardo EM, Fernández M, Pardo ML (2001). Eficiencia de las explotaciones lecheras cordobesas: análisis técnico-económico y propuestas para mejorar la rentabilidad. Ed. Analistas Económicos de Andalucía, Málaga. 144 pp. Fundación Unicaja.

Piot-Lepetit I, Vermersch D (1998). Pricing organic nitrogen under the weak disposability assumption: an application to the French pig sector. Journal of Agricultural Economics, 49(1): 85-99.

RECAN (2009) Ministerio de Agricultura, Alimentación y Medio Ambiente. Red Contable Agraria Nacional, 2009.

Ribas A, López C, Flores G (2006). Análisis no paramétrico de la eficiencia técnica de las explotaciones lecheras en Galicia: el papel de la concentración parcelaria. Revista española de estudios agrosociales y pesqueros, 209: 111-134.

Sharma KR, Leung PS, Zaleski HM (1997). Productive efficiency of swine industry in Hawaii: stochastic frontier vs. data envelopment analysis. Journal of Productivity Analysis, 8(4): 447-459.

Sharma K R, Leung P, Zaleski H M (1999). Technical, allocative and economic efficiencies in swine production in Hawaii: a comparison of parametric and nonparametric approaches. Agricultural Economics, 20(1): 23-35.

Tamminga S (2003). Pollution due to nutrient losses and its control in European animal production. Livestock Production Science, 84(2): 101-111.

Yang CC, Hsiao CK, Yu MM (2008). Technical efficiency and impact of environmental regulations in farrow-to finish swine production in Taiwan. Agricultural Economics, 39(1): 51-61.

Yang CC (2009). Productive efficiency, environmental efficiency and their determinants in farrow-to-finish pig farming in Taiwan. Livestock Science, 126(1): 195-205.

(Aceptado para publicación el 18 de septiembre de 2017) 